\title{
Methods for the Rapid Screening of Group A Streptococci: Fluorescent in situ Hybridization versus Immunochromatography
}

\author{
Jin-Ya Ding ${ }^{\text {a }}$ Pei Wang ${ }^{b}$ \\ Division of Clinical Microbiology, Department of Laboratory Medicine, a Wuhan General Hospital of Guangzhou \\ Military Command, Wuhan, and ${ }^{\mathrm{b}}$ The First People's Hospital of Jingmen, Jingmen, China
}

\section{Key Words}

Fluorescence in situ hybridization · Group A streptococci •

Streptococcal pharyngitis $\cdot$ Immunochromatographic assay

\begin{abstract}
Objective: To evaluate the accuracy of detection for screening group A streptococci (GAS) in pediatric clinics using fluorescent in situ hybridization (FISH) and immunochromatographic assay (ICA). Subjects and Methods: A group of 630 children who attended an outpatient clinic with signs and symptoms of acute upper respiratory tract infection were enrolled in this study. Specimens were collected using a double-swab collection device. Culturing methods were employed as the gold standard for comparison. Discordant results (i.e., positive results for FISH or ICA along with negative culture results) were further evaluated by using Todd-Hewitt broth (THB) with subsequent subculture for group A selective streptococcus agar. True-positive or false-positive of GAS was determined by the presence or absence of THB subculture. The diagnostic characteristics of FISH and ICA were assessed. Results: After discrepant analysis, the sensitivity, specificity and positive and negative predictive values were as follows: 89.2, 100, 100 and 95.4\%, respectively, for FISH;
\end{abstract}

corresponding values for ICA were 76.8, 98.6, 96.1 and $90.5 \%$. Conclusion: The results demonstrated that the FISH assay had a higher detection sensitivity than ICA and is suitable for rapid and accurate GAS screening in pediatric clinics.

Copyright $\odot 2011$ S. Karger AG, Basel

\section{Introduction}

Group A streptococcus (GAS), Streptococcus pyogenes, is a major cause of upper respiratory tract infections, with GAS being isolated from about $30 \%$ of children with acute pharyngitis [1]. The signs and symptoms of pharyngitis caused by GAS are nonspecific, and therefore deciding on the proper clinical diagnosis and treatment can be difficult for physicians. Accurate and prompt diagnosis of GAS is vital for optimizing antimicrobial therapy, shortening the duration of the illness, and preventing suppurative and nonsuppurative complications.

Traditionally, the gold standard for diagnosis of GAS has involved recovery of organisms from specimens by culturing, followed by biochemical analysis. This method takes at least $24 \mathrm{~h}$ to complete from the first indication of growth. In acute cases, rapid antigen detection (RAD)

\section{KARGER \\ Fax +4161306 1234 \\ E-Mail karger@karger.ch}

www.karger.com
(C) 2011 S. Karger AG, Basel

1011-7571/11/0206-0504\$38.00/0

Accessible online at:

www.karger.com/mpp 
has become a routine point-of-care test [2], and is widely used in many clinical institutions. In theory, RAD allows practitioners to accurately diagnose and treat GAS pharyngitis and thus avoid prescribing unnecessary antibiotics for viral infections [3]. Although many reports support the use of culturing and RAD for GAS diagnosis, these methods yield variable results depending upon the type of test, proficiency of personnel performing the procedure, and patient history [2]. Mirza et al. [4] reported that the sensitivity of RAD varies considerably, from 70 to $90 \%$, with the majority having a high specificity $(95 \%$ or greater). Nissinen et al. [5] recommended that external quality assurance samples be used as a tool to improve and validate the quality of RAD. Recently, fluorescent in situ hybridization (FISH) of whole cells with a fluorescence-labeled, 16S rRNA-targeted oligonucleotide probe was introduced as a diagnostic tool for the sensitive detection of microorganisms directly from clinical specimens [6-10], which resulted in an improvement in sensitivity over previous methods. The FISH method is rapid and reliable for the detection of GAS [2], and could potentially complement conventional, culture-based detection methods. On the other hand, the immunochromatographic assay (ICA) technique is simple, rapid and based on commercially available information (Inverness Medical Professional Diagnostics, Bedford, UK): the ICA for GAS detection is $95.1 \%$ sensitive and $99 \%$ specific. In our study, we compared FISH and ICA with standard culture methods, and explored their applicability for the rapid screening of GAS in throat swabs from pediatric outpatients.

\section{Subjects and Methods}

\section{Study Population and Sample Collection}

This study was conducted in two pediatric outpatient clinics of two medical centers, Wuhan General Hospital of Guangzhou Military Command and The First People's Hospital of Jingmen, China from January 2008 to March 2009. Consent was obtained from each participant and Research Ethics Board approval was obtained for the study. A group of 630 children (381 boys, 249 girls; age range from 6 months to 14 years) admitted with signs and symptoms of acute upper respiratory infection were enrolled in the study. Physicians collected a pharyngeal specimen with a double-swab collection-transport device containing $2 \mathrm{ml}$ of liquid Amies medium in a sponge-like pledget (Copan, Corona, Calif., USA). One swab was used for FISH, the second was used for ICA (Clearview Exact Strep A, UK). The pledget was placed in the collection device and used to inoculate $5 \%$ sheep blood agar plates, which were then incubated with $5-10 \% \mathrm{CO}_{2}$ for $24 \mathrm{~h}$ at $37^{\circ} \mathrm{C}$. All specimens were tested in a blinded manner.

\section{Oligoprobes}

One probe was chosen from the literature for the detection of GAS, S. pyogenes probe (SPY probe) [9], and a second probe (EUB338 probe) was also chosen from the literature to detect the presence of the bacteria [11]. The sequence of the probes was as follows: SPY probe, 5'-TTC CAA AGC GTA CAT TGG TT-3', with the $5^{\prime}$ end linked to fluorescein isothiocyanate; and EUB338 probe, 5'-GCT GCC TCC CGT AGG AGT-3', with the 5' end linked to cyanine dye 3 . The EUB338 probe was used as a positive control. Probes were synthesized by EuroGentec BV (Maastricht, The Netherlands).

\section{FISH Assay}

The FISH assay was performed as previously described by Kempf et al. [9] with the following modifications: briefly, one swab was used to make a smear onto glass slides, which were flamed with a Bunsen burner for several seconds. Gram-positive streptococci were permeabilized by incubating the fixed slide with lysozyme $\left(1 \mathrm{mg} / \mathrm{ml}\right.$ for $10 \mathrm{~min}$ at $\left.30^{\circ} \mathrm{C}\right)$ dissolved in $10 \mathrm{mM}$ Tris ( $\mathrm{pH}$ 8.0). The reaction was stopped by rinsing the slide thoroughly with Milli-Q water. Then, $100 \mu$ l of each oligonucleotide probe $(10 \mathrm{ng} / \mathrm{ml})$ was added to the slides and hybridized at $46^{\circ} \mathrm{C}$ for $60 \mathrm{~min}$ using a $20 \mathrm{~mm}$ Tris- $\mathrm{HCl}$ buffer $(20 \mathrm{~mm}$ Tris- $\mathrm{HCl}$, $0.9 \mathrm{M} \mathrm{NaCl}, 0.1 \%$ SDS, $30 \%$ formamide, $\mathrm{pH}$ 7.2). After hybridization, the slides were washed for $10 \mathrm{~min}$ at $46^{\circ} \mathrm{C}$ in a washing buffer (20 mM Tris-HCl, $0.9 \mathrm{M} \mathrm{NaCl}, \mathrm{pH} 7.2$ ) and mounted with VectaShield (Vector Laboratories, Burlingame, Calif., USA). The slides were immediately evaluated by epifluorescence microscopy (Olympus BX40, Japan). The SPY probe was applied simultaneously with the EUB338 probe on the same slide.

\section{Immunochromatographic Assay}

ICA was performed according to the manufacturer's instructions (Clearview Exact Strep A). The results were read at 5 min and interpreted by noting the presence or absence of visually detectable pink to purple lines.

\section{Culturing and Identification of Organisms}

The pledget of the collection device was removed with sterile forceps to inoculate a $5 \%$ sheep blood agar plate, which was then incubated with $5-10 \% \mathrm{CO}_{2}$ for $24 \mathrm{~h}$ at $37^{\circ} \mathrm{C}$. Colonies presumed to be beta-hemolytic streptococci were confirmed by achieving Gram staining and translucent colonies with a zone of hemolysis, bacitracin susceptibility, and a latex agglutination test (BioMérieux, France).

\section{Definitions and Discordant Results}

A true-positive result was defined as being culture-positive for GAS or as both FISH and ICA yielding positive results consistent with GAS. A true-negative result was defined as a specimen which tested negative for GAS by all methods. With discordant results (only FISH or ICA positive), GAS was confirmed using additional throat swab samplings, which were placed in a vial of ToddHewitt broth (THB)-selective enrichment broth $(5 \mathrm{ml}, \mathrm{BBL}$, Cockeysville, Md., USA) with $5-10 \% \mathrm{CO}_{2}$ for $18-24$ h at $37^{\circ} \mathrm{C}$, then subcultured on selective streptococcus agar (SSA) plates for purity. A discordant sample that was positive by THB/SSA culture was defined as a true-positive result; a discordant sample that was negative by THB/SSA culture was defined as a false-positive result. 
Table 1. Comparison of FISH and ICA methods for the detection of GAS

\begin{tabular}{|c|c|c|c|c|}
\hline \multirow[t]{2}{*}{ Culture } & \multicolumn{2}{|l|}{ FISH } & \multicolumn{2}{|l|}{ ICA } \\
\hline & positive & negative & positive & negative \\
\hline Positive & 165 & 21 & 146 & 40 \\
\hline Negative & 8 & 436 & 9 & 435 \\
\hline
\end{tabular}

\section{Results}

\section{Diagnostic Characteristics of the Two Detection} Methods

Of the 630 samples tested, 186 (29.5\%) were GAS-positive by culturing. Eight additional samples were defined as positive by FISH or ICA but not by culturing. Of the 8 samples, 3 were defined as positive without discrepancy analysis since they were both FISH- and ICA-positive; 5 were defined as positive after discrepancy analysis since they were either FISH- or ICA-positive. The performance of FISH and ICA testing compared to culturing is shown in tables 1 and 2. In this study, good levels of sensitivity $(89.2 \%)$ and specificity (100\%) were achieved by FISH compared with culture, and the diagnostic value of FISH is superior to that of ICA for the detection of GAS.

\section{Discrepancy Analysis}

A discordant result was obtained for 11 samples. In these cases, a positive result was obtained for either FISH or ICA along with a negative culture result. The results of discrepancy analysis are shown in table 3.

\section{Discussion}

Our findings indicated that FISH was more sensitive than ICA, but FISH was less sensitive than culturing. Among our specimens, 21 were found to be FISH-negative but culture-positive. A culture of a pure colony of GAS was tested by FISH. Further investigation confirmed that all colonies were FISH-positive, thereby revealing that the FISH probe may miss the GAS strain in the swab, because the low number of bacterial organisms in the swab was undetectable by FISH assay. Culturing was more time-consuming and more expensive than FISH. Interestingly, eight specimens were culture-negative but FISH-positive. By checking the patients' medical records, five of these eight patients were found to have suffered
Table 2. Diagnostic value of FISH and ICA methods for the detection of GAS after discordant analysis

\begin{tabular}{lllll}
\hline Assay & Sensitivity, \% & Specificity, \% & PPV, \% & NPV, \% \\
\hline FISH & $89.2(173 / 194)$ & $100(436 / 436)$ & $100(173 / 173)$ & $95.4(436 / 457)$ \\
ICA & $76.8(149 / 194)$ & $98.6(430 / 436)$ & $96.1(149 / 155)$ & $90.5(430 / 475)$ \\
\hline \multicolumn{2}{c}{ PPV = Positive predictive value; NPV = negative predictive value. } \\
\hline
\end{tabular}

from GAS infection previously and to have been administered a course of antibiotics before attending the clinic. These findings indicated that the FISH assay can detect organisms treated antibiotics with a lower fluorescence signal, as reported by Hogardt et al. [10]. There are currently no reported studies describing the effect of antibiotic treatment on the life span of the rRNA target for GAS, and future studies should be carried out to investigate this. Unlike ICA, no false-positive results (samples positive by ICA yet negative both by culture and FISH) were obtained in the FISH assay, which could have important implications for GAS screening in pediatric clinics, as it could prevent patients from receiving unnecessary antibiotic therapy.

The cell wall of GAS is easily penetrated by oligoprobes and an intense hybridization signal can be observed within $30 \mathrm{~min}$. The sensitivity of FISH is lower than the direct probe test reported by Chapin et al. [2], but the morphology of GAS cells is maintained during the FISH assay, which was helpful for diagnosis, whereas cell morphology is not available for the direct probe test. Polymerase chain reaction (PCR) is also more sensitive than the FISH assay; however, the PCR assay has stringent space requirements and is more expensive than FISH, and the interpretation of PCR results can be difficult with regard to clinical decision making because PCR detects all bacterial DNA present in the throat swab, including dead or degraded bacteria as well as living bacteria. Few specimens have problems with background fluorescence, but even so, it is easy to discriminate the intense signal of GAS from background fluorescence. In contrast to a peptide nucleic probe (PNA), a DNA probe requires enzymatic pretreatment. Nevertheless, the advantages of using a DNA probe compared to a PNA probe are lower cost and flexibility in the design and synthesis of the probes. Equally important, until the present time, the description of the PNA probe for GAS is not available in the literature. 
Table 3. Discrepancy analysis of 11 samples by THB/SSA culture

\begin{tabular}{|c|c|c|c|c|c|}
\hline Case & FISH & ICA & $\begin{array}{l}\text { SBA } \\
\text { culture }\end{array}$ & $\begin{array}{l}\text { THB/SSA } \\
\text { culture }\end{array}$ & $\begin{array}{l}\text { Test result } \\
\text { interpretation }\end{array}$ \\
\hline 1 & positive & negative & negative & positive & true positive (FISH) \\
\hline 2 & positive & negative & negative & positive & true positive (FISH) \\
\hline 3 & positive & negative & negative & positive & true positive (FISH) \\
\hline 4 & positive & negative & negative & positive & true positive (FISH) \\
\hline 5 & positive & negative & negative & positive & true positive (FISH) \\
\hline 6 & negative & positive & negative & negative & false positive (ICA) \\
\hline 7 & negative & positive & negative & negative & false positive (ICA) \\
\hline 8 & negative & positive & negative & negative & false positive (ICA) \\
\hline 9 & negative & positive & negative & negative & false positive (ICA) \\
\hline 10 & negative & positive & negative & negative & false positive (ICA) \\
\hline 11 & negative & positive & negative & negative & false positive (ICA) \\
\hline
\end{tabular}

All cases had been treated with antibiotics before visiting the clinic.

RAD tests are frequently used to detect GAS pharyngitis. The sensitivity of ICA measured in this study was $76.8 \%$, similar to that previously reported by Mirza et al. [4]. Six false-positive results were obtained with ICA in our study (table 3) and all of these false-positive results were both culture- and FISH-negative. False-positive results may be associated with the presence of cross-reactive antigens between GAS and other beta-hemolytic streptococci. The medical records of the patients with false-positive results showed that they had been treated with antibiotics. Three samples were ICA- and FISH-positive but culture-negative, and the reason for this may be that ICA was able to detect the antigen of dead GAS, while FISH can detect strains of nonculturable bacteria that are dormant but not dead, that may still be pathogenic [12]. Our results indicated that the sensitivity of FISH was higher than that of ICA (89.2 vs. $76.8 \%)$, and that antibiotic usage or the proportion of GAS in a dormant state did not affect the performance of the FISH test. However, ICA was not useful for patients with suspected GAS antibiotic treatment failure and subsequent reinfection [2].

Five cases that were FISH-positive and sheep blood agar culture-negative were defined as GAS-positive on the basis of THB/SSA results, indicating that the recovery of GAS was enhanced by THB/SSA culture. The strains missed by sheep blood agar culture may be attributed to the presence of a nonhemolytic GAS phenotype, or the inhibition of GAS by rapidly growing microorganisms, including beta-hemolytic bacteria, such as Staphylococcus aureus. Because discrepancy analysis was based on the culture method rather than on molecular assay, the results may not be $100 \%$ reliable. Even if the cultures are taken as the single gold standard, the FISH data in this study would still be convincing for rapid screening of GAS.

\section{Conclusion}

The data showed that the FISH assay was more sensitive than ICA and therefore a more accurate and reliable method for the rapid screening of GAS in pediatric clinics. This methodology may reduce the unnecessary use of antibiotics for pediatric patients with acute upper respiratory tract infection.

References

1 Hall MC, Kieke B, Gonzales R, Belongia EA: Spectrum bias of a rapid antigen detection test for group A beta-hemolytic streptococcal pharyngitis in a pediatric population. $\mathrm{Pe}$ diatrics 2004;114:182-186.

$\checkmark 2$ Chapin KC, Blake P, Wilson CD: Performance characteristics and utilization of rapid antigen test, DNA probe, and culture for detection of group A streptococci in an acute care clinic. J Clin Microbiol 2002;40:42074210.

- 3 Sheeler RD, Little P: Rapid streptococcal testing for sore throat and antibiotic resistance. Clin Microbiol Infect 2006;12 (suppl):3-7.

4 Mirza A, Wludyka P, Chiu TT, Rathore MH: Throat culture is necessary after negative rapid antigen detection tests. Clin Pediatr (Phila) 2007;46:241-246. 
5 Nissinen A, Strandén P, Myllys R, Takkinen J, Björkman Y, Leinikki P, Siitonen A: Pointof-care testing of group A streptococcal antigen: performance evaluated by external quality assessment. Eur J Clin Microbiol Infect Dis 2009;28:17-20.

6 Trebesius K, Leitritz L, Adler K, Schubert S, Autenrieth IB, Heesemann J: Culture independent and rapid identification of bacterial pathogens in necrotising fasciitis and streptococcal toxic shock syndrome by fluorescence in situ hybridisation. Med Microbiol Immunol 2000;188:169-175.
7 Poppert S, Nickel D, Berger A, Yildiz T, Kaestner N, Mauerer S, Spellerberg B: Rapid identification of beta-hemolytic streptococci by fluorescence in situ hybridization (FISH). Int J Med Microbiol 2009;299:421-426.

$>8$ Tavares A, Inácio J, Melo-Cristino J, Couto I: Use of fluorescence in situ hybridization for rapid identification of staphylococci in blood culture samples collected in a Portuguese hospital. J Clin Microbiol 2008;46:3097-3100.

-9 Kempf VA, Trebesius K, Autenrieth IB: Fluorescent in situ hybridization allows rapid identification of microorganisms in blood cultures. J Clin Microbiol 2000;38:830-838.

10 Hogardt M, Trebesius K, Geiger AM, Hornef M, Rosenecker J, Heesemann J: Specific and rapid detection by fluorescent in situ hybridization of bacteria in clinical samples obtained from cystic fibrosis patients. J Clin Microbiol 2000;38:818-825.
1 Amann RI, Ludwig W, Schleifer KH: Phylogenetic identification and in situ detection of individual microbial cells without cultivation. Microbiol Rev 1995;59:143-169.

12 Waar K, Degener JE, van Luyn MJ, Harmsen HJ: Fluorescent in situ hybridization with specific DNA probes offers adequate detection of Enterococcus faecalis and Enterococcusfaecium in clinical samples. J Med Microbiol 2005;54:937-944. 